\title{
Afrikanische Silhouetten und Feldfotografie
}

\section{Griaules Beitrag zur Mausschen „Entdeckung“ der Körpertechniken}

Philippe Despoix

African Silhouettes and Field Photography. M. Griaule's Contribution to the Maussian "Discovery" of Body Techniques

This essay focuses on the interaction between the new reproduction media and corresponding reconfiguration of research fields in anthropology using the case of the "techniques of the body" - a concept developed by Marcel Mauss (1872-1950). For Mauss, the initiator of this discipline in France, body skills constituted the most important anthropological entity resulting from the confrontation of technical images and his interest in walking techniques. Three scenarios are especially significant for Mauss's formulation of "body techniques" as a genuine concept: the front during the World War I, a New Yorker hospital in 1926, and an ethnographical field study conducted in Africa during the late 1920s. Both, the photographic media as well as the Abyssinian expedition of his student Marcel Griaule, whose research publication Mauss co-authored (Silhouettes et graffiti abyssins) in 1933, take centre stage here.

Keywords: Photographic media, silhouette, body, technique, cultural anthropology

Schlüsselwörter: Fotografische Medien, Silhouette, Körper, Technik, Ethnologie

Marcel Mauss, seit 1901 Mitarbeiter der Zeitschrift Année Sociologique, 1925 Mitbegründer des Institut d'Ethnologie und ab 1931 Professor am College de France, verfügte über ein legendäres Wissen als Anthropologe, das vor allem auf Schrift- und Buchgelehrsamkeit beruhte. Insofern scheinen auf den ersten Blick seine Arbeiten auf Feldforschung basierender Ethnologie eher entgegengesetzt zu sein. Außer seinen Studienaufenthalten (in Holland und England) und seiner Mobilisierung an die Front im Ersten Weltkrieg ging er nur zweimal auf größere Auslandsreisen: die erste führte ihn 1926 in die USA, die zweite 1930 für einen kurzen Aufenthalt nach Marokko. Doch obgleich diese Erfahrungen jenseits der akademischen Welt deutliche Ausnahmen in seiner intellektuellen Biographie darstellen, bilden sie möglicherweise 
- zusammen mit der Anteilnahme an den Expeditionen seines Schülers Marcel Griaule - die ausschlaggebenden Zäsuren für die wichtigste Entdeckung seines Spätwerkes: die techniques du corps, die „Körpertechniken“.

\section{Mauss' Mediale Reminiszenz und die Gangtechnik}

Der Begriff wurde von Mauss zum ersten Mal 1934 öffentlich in einem Vortrag entwickelt, den er auf Einladung der französischen Société de psychologie hielt (Mauss [1936] 1950). Die Hauptthese ist so einfach wie radikal: Die Technik beginnt für den Menschen nicht erst mit dem Werkzeug, sondern viel eher, nämlich mit dem Körper selbst. Daraus ergibt sich für den Anthropologen die Notwendigkeit, alle möglichen Formen von Körpertechniken zu studieren: unabhängig davon, ob der Körper allein im Spiel oder ob dieser mit einem Werkzeug oder sogar einer Maschine verbunden ist. Es ist charakteristisch für Mauss, dass er bei diesem Vortrag nicht bloß seine These darlegte, sondern gleichzeitig die Umstände ihrer Entdeckung und die Etappen ihrer begrifflichen Formulierung erläuterte. Die erste lebensweltliche Situation, in der ihm die kulturelle Eigenart elementarer Techniken aufging, war die Fronterfahrung. Mauss zog zunächst als dolmetschender Offizier der 27. britischen Division in den Ersten Weltkrieg. Diese Vermittlerfunktion zwischen den britischen und französischen Truppen scheint ein besonders geeigneter Posten gewesen zu sein, um die „Übertragbarkeit“ nicht allein von Befehlen, sondern der alltäglichsten Gesten zu beobachten, wie - so sein erstes Beispiel das Ausheben der Schützengräben. Die britischen Soldaten konnten nämlich nicht die Spaten der Franzosen bedienen und umgekehrt. So war man aufgrund der unterschiedlichen Techniken des Grabens jedes Mal gezwungen, wenn eine französische Division die englischen Truppen ablöste, die verwendeten Spaten auszuwechseln. Da es sich dabei immerhin um 8000 Stück handelte, wurde das unbemerkte Phänomen plötzlich sichtbar (ebd.: 366).

Mauss' Grundgedanke konzentrierte sich aber in dem Vortrag auf eine der elementarsten menschlichen Aktivitäten, die sich durch eine fast völlige Abwesenheit von Hilfsmitteln auszeichnet: das Gehen, und zwar zunächst in der Form des Marschierens, das für ihn eine Technik im vollen Sinne des Wortes darstellte. Sofern sein Rhythmus ein Element des Zusammenhalts der Gruppe ist, verweist es auf ein eigentlich „soziales“ Phänomen. So heißt es im bewusst anekdotisch gehaltenen Bericht, in dem Mauss seine Überlegung schilderte:

Sie wissen alle, dass die britische Infanterie in einem anderen Schritt als die französische marschiert. [...] Das Regiment von Worcester, das während der Schlacht an der Aisne Seite an Seite mit der französischen Infanterie bedeutende 
Geländegewinne gemacht hatte, bat um die königliche Erlaubnis, französische Schellen und Schlagzeuge und eine Gruppe französischer Trompeter und Trommler einsetzen zu dürfen. Das Resultat war entmutigend. Fast sechs Monate lange sah ich in den Straßen von Bailleul, lange nach der Schlacht [...], folgendes Schauspiel: das Regiment hatte seinen englischen Marschschritt beibehalten und passte ihn dem französischen Takt an. [...] Das unglückliche Regiment der großen Engländer konnte nicht defilieren. Der Marsch war ein einziger Missklang. Wenn das Regiment im Gleichschritt zu marschieren versuchte, stimmte die Musik nicht mit dem Schritt überein. Daher war [es] gezwungen, seine französischen Marschklänge aufzugeben. (Mauss [1936] 1950: 367, [1936] 1975: 201) ${ }^{1}$

Die Szene erinnert an einen Film-Slapstick. Angesichts des wiederholten, grotesken Scheiterns jeder Synchronisierung zwischen dem britischen und dem französischen Gleichschritt wird dem Anthropologen deutlich, dass auch die elementarsten Techniken auf ,kollektive‘ Weise angeeignet werden. Sogar das Gehen besitzt seine spezifischen Formen, und das ihnen gemeinsame Element findet sich in den erlernten Rhythmen des Körpers, die den Gang strukturieren. Die Gangart sowie auch umgekehrt die Techniken des SichAusruhens werden so in jener ,interkulturellen' Ausnahmesituation des Krieges beobachtet, zum neuen Gegenstand der Anthropologie.

Man mag es erstaunlich finden, dass Mauss an dieser Stelle die chronofotografischen Arbeiten von Marey oder Muybridge über die menschliche Bewegung aus den 1880er und 90er Jahren nicht erwähnt hat, oder dass er sich nicht auf die vergleichenden Aufnahmen verschiedener Gangarten stützte, die Félix Regnault im Anschluss an Marey gemacht hat. Regnault hatte bereits 1895, anlässlich der ethnographischen Ausstellung über Westafrika auf dem Pariser Champ de Mars, unter anderem gehende und rennende Afrikaner chronofotografisch festgehalten (Comte/Regnault 1896: 337-341, Braun 2006: 75 f.). Wenig später nahm er den Chef d'escardron des 34. französischen Artillerie-Regiments, Albert de Raoul, beim Gehen und im sogenannten "gebeugten Lauf" (allure en flexion) auf. De Raoul ahmte die entsprechende afrikanische Gehtechnik mit dem ausdrücklichen Ziel nach, die Leistungen des französischen Soldaten zu verbessern. Darüber hatte Regnault 1896 in den Bulletins der Société d'anthropologie de Paris berichtet (vgl. Abb. 1).

Die Blütezeit der Marey'schen Chronofotografie lag zwar um 1900, doch Mauss muss diese Technik gekannt haben. Er erwähnte sie aber weder in seinem Vortrag noch in seinen anderen Schriften.

Es war die Begegnung mit der amerikanischen Kultur und einem anderen technischen Medium, die es Mauss etwa zehn Jahre später ermöglichte, das Potential seiner sonderbaren Beobachtungen an der Front zu erschließen. Auf Einladung der Rockefeller Foundation weilte der Anthropologe nach einer Periode starker beruflicher Belastung - das Pariser Institut für Ethnologie war gerade gegründet worden - im Mai 1926 in New York. Der New Yorker Aufenthalt bildete die erste Etappe einer Amerika-Tournee, die ihn an die 

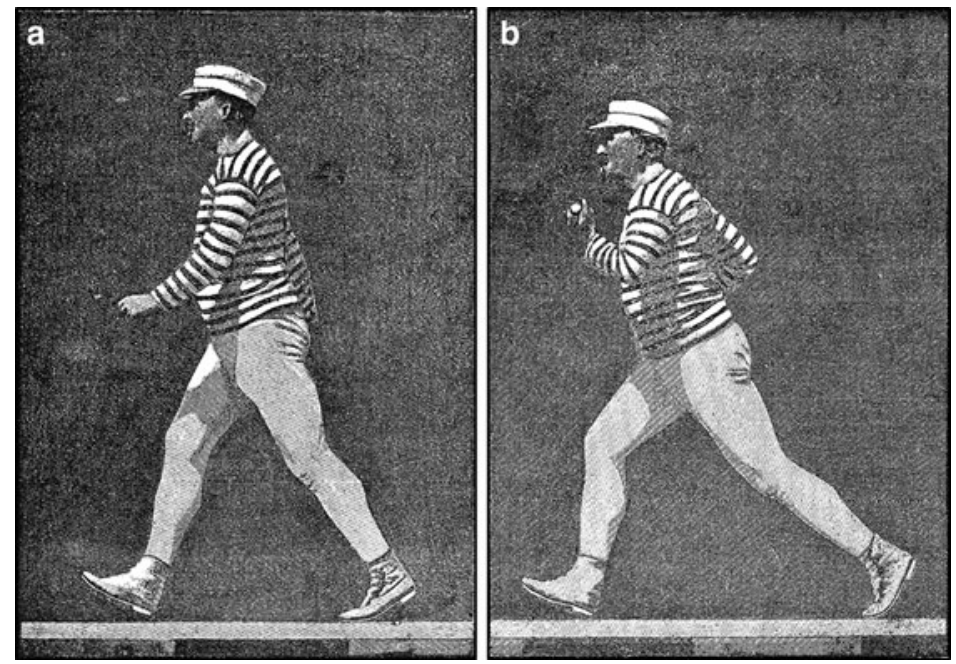

Abb. 1 Darstellung des ,gewöhnlichen“ Gangs (links) und des „gebeugten“ Laufs (rechts) (Comte/Regnault 1896, 338 f.)

großen Universitäten führen sollte, um von Franz Boas bis Ezra Park die führenden Kollegen seiner Disziplin zu treffen und verschiedene Seminare abzuhalten. Doch kaum angekommen erkrankte der erschöpfte Mauss wahrscheinlich an Dysenterie - und sah sich mit Arbeitsverbot an ein Krankenhausbett gefesselt. ${ }^{2}$ Die autobiographische Anekdote, die er in diesem Zusammenhang im Vortrag von 1934 erzählte, ist entscheidend:

Eine Art Erleuchtung kam mir im Krankenhaus. Ich war krank in New York. Ich fragte mich, wo ich junge Mädchen gesehen hatte, die wie meine Krankenschwestern gingen. Ich hatte genug Zeit, darüber nachzudenken. Ich fand schließlich heraus, dass es im Kino gewesen war. Nach Frankreich zurückgekehrt, bemerkte ich vor allem in Paris die Häufigkeit dieser Gangart; die jungen Mädchen waren Französinnen und gingen doch in dieser Weise. In der Tat begann die amerikanische Gangart durch das Kino bei uns verbreitet zu werden. Das war ein Gedanke, den ich verallgemeinern konnte. Die Stellung der Arme, der Hände während des Gehens, stellen eine soziale Eigenheit dar und sind nicht einfach ein Produkt irgendwelcher rein individueller, fast ausschließlich psychisch bedingter Abläufe und Mechanismen. (Mauss [1936] 1950: 368, [1936] 1975: 202)

Der Ausdruck „Erleuchtung“ (révélation) bezeichnet hier einen paradoxen Erkenntnisprozess, der selten kommentiert wird. ${ }^{3}$ Anstelle eines wissenschaftlichen Experiments beruft sich Mauss auf eine „unwillkürliche“ Erinnerung: zunächst an Kinobilder, die vor seinem inneren Auge aufziehen. Diese wurde durch zwei sich überlagernde Eindrücke ausgelöst: das aktuelle Bild der amerikanischen Krankenschwestern und das einst auf einer Pariser 
Kino-Leinwand Gesehene. Und schließlich das Aufblitzen des singulären, aber deutlich erkennbaren Charakters ihrer Gangart. Die Wahrnehmung des Unterschieds in jener Gehtechnik scheint nur a posteriori, auf dem Weg des Wiedererkennens, konstatiert und auf den Begriff gebracht werden zu können. Das Paradoxon einer solchen „epistemischen Entdeckung“, die sich außerhalb jeder beruflichen Arbeit ereignet, entspricht auch dem Gegensatz zwischen der liegenden, inaktiven Position des männlichen Beobachters und dem Akt des Gehens der weiblichen Krankenpflegerinnen. Es ist sozusagen ein vielfältiger Effekt der Distanzierung: verordnete Bettruhe, Entfernung vom vertrauten Pariser akademischen Milieu und schließlich das filmische Wiedererinnern. Genau diese kinematografisch vermittelte,Verfremdung bildete den Ursprung der unwillkürlichen Assoziationskette, an deren Ende das reflexive Konzept der Körpertechniken stand. ${ }^{4}$

Die filmische Erinnerung, die Mauss ohne nähere Präzisierung erwähnt, lässt sich mit Sicherheit in die erste Hälfte der Zwanziger Jahre zurückdatieren. Die Nachkriegszeit ist die Blütezeit des Stummfilms, dessen Ästhetik sich auf die menschliche Physiognomie konzentrierte und dessen visuelle Potenz nicht durch Sprache eingeschränkt war. Es ist zugleich die Zeit, in der das amerikanische Kino das europäische Massenpublikum zu erobern begann. Dies waren also ideale Bedingungen für das „Offenbarungspotential“ des neuen Mediums, seine spezifische Eignung, Unterschiede sichtbar zu machen. ${ }^{5} \mathrm{Um}$ der Distanz zwischen den Kontinenten und ihren Körpertechniken gewahr zu werden, bedurfte es freilich der Gelegenheit, die amerikanische Gangart, gesehen in Filmen in Paris, mit dem Gang der New Yorker Frauen in Wirklichkeit vergleichen zu können.

Auf die neue Bedeutung einer allgemeinen technischen Vermittlung hatte im übrigen der Anthropologe schon 1931 in seinem Vortrag über Les civilisations hingewiesen: weil das filmische Medium begann, universell zu werden und die alltäglichen Attribute, die jeder Kultur zueigen waren, überall zu verbreiten, war es nun möglich, deren schnelle Veränderung und vorher nicht sichtbaren Unterschiede in Raum und Zeit wahrzunehmen (Mauss [1930] 1968: 250 f.). Man kennt auch die Schlussfolgerung, die Mauss in seiner nächsten Vorlesung über die Technologie 1935-1936 für die ethnographische Feldforschung zog: „Das Ensemble der Körperhaltungen ist eine Technik, die gelehrt wird. [...] Die[se] Körpertechniken sollen mithilfe der Fotografie und, wenn möglich, der filmischen Zeitlupe untersucht werden" ${ }^{6}$ So wird das Reproduktionsmedium, das den kulturbedingten Charakter des menschlichen Gehens offenbart hatte, zum Untersuchungs- und Analysewerkzeug für die Gesamtheit der Techniken des Körpers. ${ }^{7}$ Indem Mauss vorschlägt, die Fotografie in Form dynamischer Serien zu verwenden sowie die Zeitlupe im Film einzusetzen, spricht er dem technischen Bildmedium eine zunächst analytische Funktion zu. Diese hatten seine Schüler, insbesondere Marcel Griaule, tatsächlich begonnen produktiv zu nutzen. 


\section{Alltagstechniken und Schattenbilder: Griaules Fotografien aus Abessinien}

Aber bereits in dem Vortrag von 1934 trieb Mauss die Ergebnisse seiner Entdeckung auf die Spitze, indem er die Beziehungen zwischen Körpertechniken, Magie und Mystik in den Blick nahm. Es ist anzunehmen, dass hierfür die direkte Erfahrung von Besessenheitstänzen bei den Gnawa, also den Anhängern des Bori-Kultes, deren Riten er während seiner kurzen Mission in Marokko 1930 beiwohnen konnte, eine gewichtige Rolle spielte. Durch diese Besessenheitsriten konnte er selber beobachten, wie die Trance als Körpertechnik - ein von Musik begleiteter Tanz - eine ekstatisch-religiöse beziehungsweise therapeutische Erfahrung unterstützen konnte. Diese einmalige ethnologische Felderfahrung in Marroko könnte Aufschluss darüber geben, wieso Mauss sein Exposé mit einer letzten Reduktion des Begriffs der Körpertechniken abschließt, die tatsächlich die Kommunikation mit den Göttern berührte. ${ }^{8}$ Nach den Techniken der Bewegung, des Ausruhens, nach der Auflistung aller möglichen Alltagstechniken kommt er dann zur determinierenden Funktion der Atmungstechniken:

Ich glaube, dass es bestimmt und sogar als Grundlage all unserer mystischen Zustände Techniken des Körpers gibt, die [...] in China und Indien ausgezeichnet studiert wurden, und zwar zu einer sehr frühen Zeit. Diese sozio-psycho-biologische Untersuchung der Mystik muss unternommen werden. [...] Wenn auch die Technik der Atmung usw. einzig in Indien und China der grundlegende Gesichtspunkt ist, so halte ich sie doch für viel weiter verbreitet. (Mauss [1936] 1950: 386, [1936] 1975: 220)

Indem er Technik als eine acte traditionnel efficace, eine „traditionelle wirkungsvolle Handlung“ definierte (Mauss [1936] 1950: 371; [1936] 1975: 205), die primär auf dem Körper und seinen Rhythmen basiert, entfernte sich Mauss von den Anschauungen, die die Anthropologie seiner Zeit dominierten wie etwa die von Frazer oder selbst die seines Kollegen Lévy-Bruhl. Beide sahen in Magie und Religion eher ein Vorläuferstadium oder eine unreife Form von technischem Wissen. Im Gegensatz dazu konstatiert Mauss jedoch in Form der „Körpertechniken“ eine deutliche Kontinuität zwischen Magie und Technik. Er sieht beide prinzipiell ineinander verflochten: „Acte technique, acte physique, acte magico-religieux sont confondus pour l'agent" - „Technische Handlung, physische Handlung, magisch-religiöse Handlung sind für den Handelnden verschmolzen“ (Mauss [1936] 1950: 371, [1936] 1975: 205). Dies ist zweifellos die Formulierung, die den radikaleren Kern seines Vortrags auf den Punkt brachte.

Diesen Kern mag ein einzigartiges Dokument erhellen, das Mauss im Vorhaben, die Körpertechniken zum zentralen Untersuchungsobjekt der Anthropologie zu erklären - auch in Betonung ihrer engen Beziehung zum magisch-religiösen Bereich - bestätigte. Es handelt sich um Bildmaterial, das 
sein Mitarbeiter Marcel Griaule 1928-1929 auf einer ethnologischen Expedition in Abessinien beim Studium der Godjan-Kultur gesammelt und 1933 unter dem Titel Silhouettes et graffiti abyssins veröffentlicht hatte. ${ }^{9}$ In diesem außerordentlichen Material wurden Alltagstechniken in Form von Schattenbildern unter einem einheimischen Gesichtspunkt dargestellt.

Obgleich diese Bilddokumente ausschließlich aus abessinischen Kirchen stammen, gilt hier das ethnographische Interesse Griaules nicht der traditionellen religiösen Kunst, sondern den umrissartigen Figurenzeichnungen oder Silhouetten auf jenen ornamentalen Friesen, die die sakralen Fresken umgeben sowie den grotesken Graffiti junger Abessinier, die an den Kapellenmauern den kleinsten verfügbaren Platz ausnutzten, um sich im Zeichnen zu üben (vgl. Abb. 2).

Die Graffiti sind zum Teil in die moderne Kunstgeschichte eingegangen, weil Griaule Beispiele davon in der surrealistischen Zeitschrift Documents, zu deren Mitarbeitern er gehörte, veröffentlichen ließ. Die Silhouetten dagegen sind mehr oder weniger unbeachtet geblieben. ${ }^{10}$ Mit der Serie fotografischer Aufnahmen, die Griaule im seinem Band publizierte, zoomte er jeweils von einer Fern- zur Nahsicht heran: vom Kirchengebäude in der Gesamtsicht zum Kircheninneren mit den großen religiösen Fresken über Aufnahmen des Malers und Dekorateurs selbst bis hin zu den Ornamentdetails der Friese, in welche die silhouetteartigen Muster eingebettet sind (Abb. 3, 4):

Der Einsatz des Mediums Fotografie trägt hier deutlich zur Enthierarchisierung der visuellen Elemente bei: Er erlaubt eine Dezentrierung der herkömmlichen Sehweise, die in religiösen Inhalten oder deren ikonographischer Form das Kennzeichen dieser ,archaischen“ Kunst gesehen hätte. Erst durch die fotografische Vergrößerung konnte die ornamentale Umrahmung, die sich an der Peripherie des hieratischen Bereichs befindet, zum eigenständigen Untersuchungsobjekt werden.

a

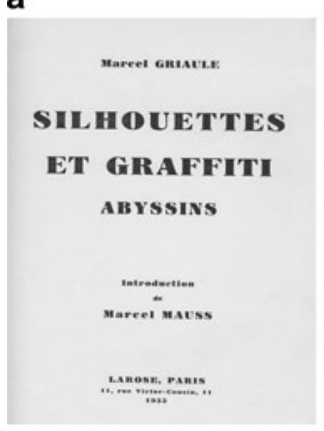

b

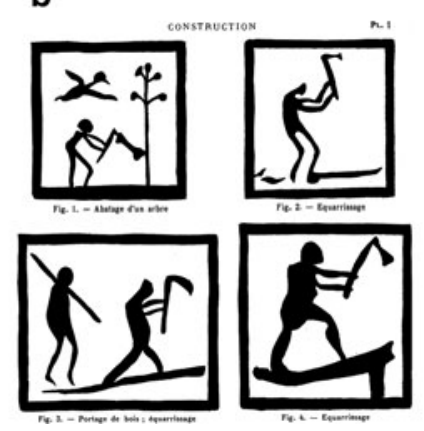

C

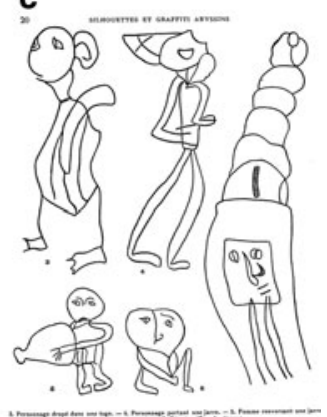

Abb. 2 a Titelblatt (Griaule 1933), b Silhouetten 1-4: Bautechnik (Griaule 1933: Bildtafel 1), c Graffiti 3-7 (Griaule 1933: 20) 

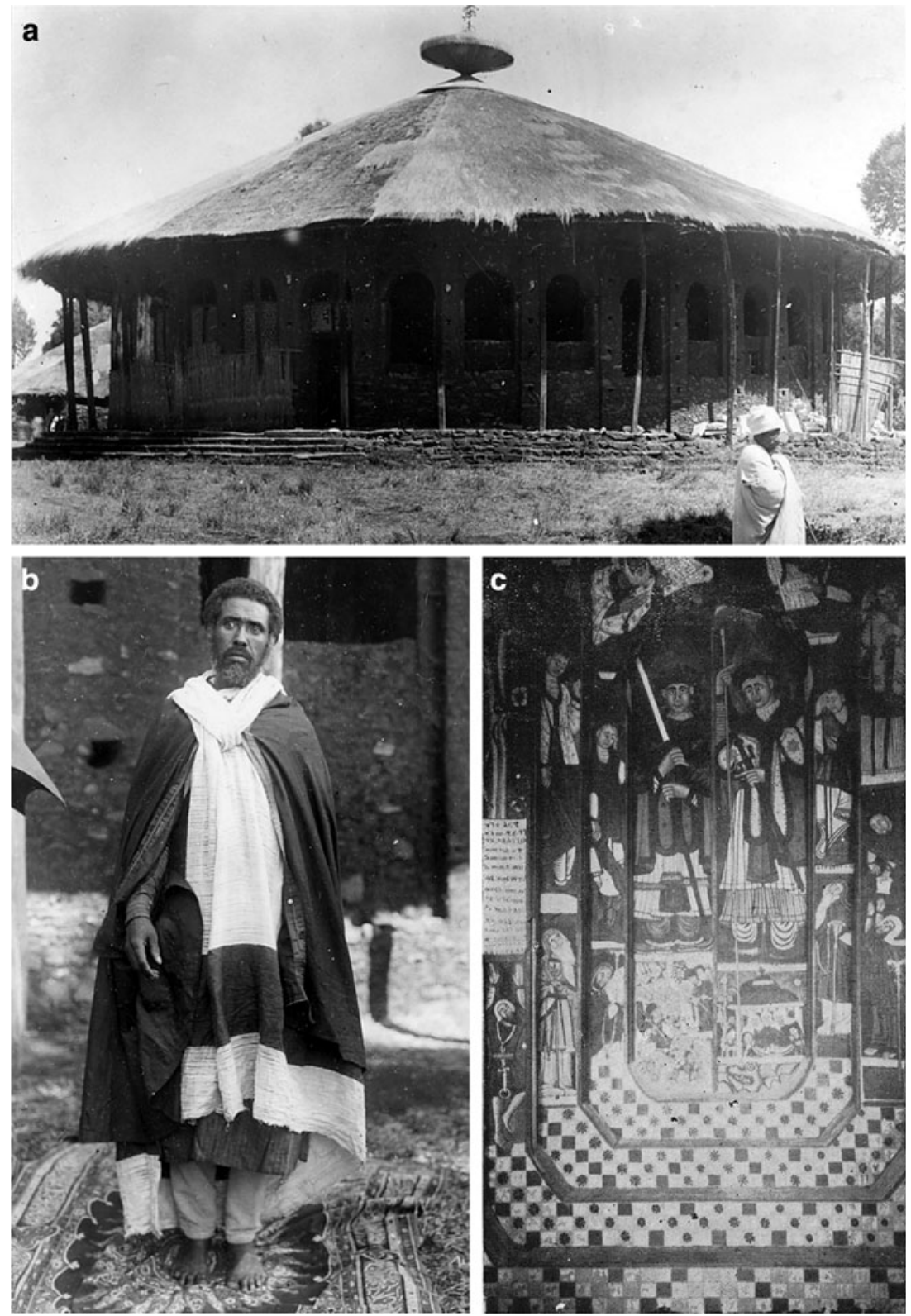

Abb. 3 a Kirche in Dabra Markos (Griaule 1933: Tafel II, Abb. 3), b Wouddié, Maler der Zarabruk Kirche (Griaule 1933: Tafel III, Abb. 7), c Westtor von Zarabruk (Griaule 1933: Tafel V, Abb. 10)

Die kleinen Silhouetten, die in den schwarzweißen, schachbrettartigen Fries hineingemalt sind, umgeben die Fresken der Szenen aus dem Alten und Neuen Testament. Ihre Hauptmotive sind „Techniken des Alltags“, die auf 

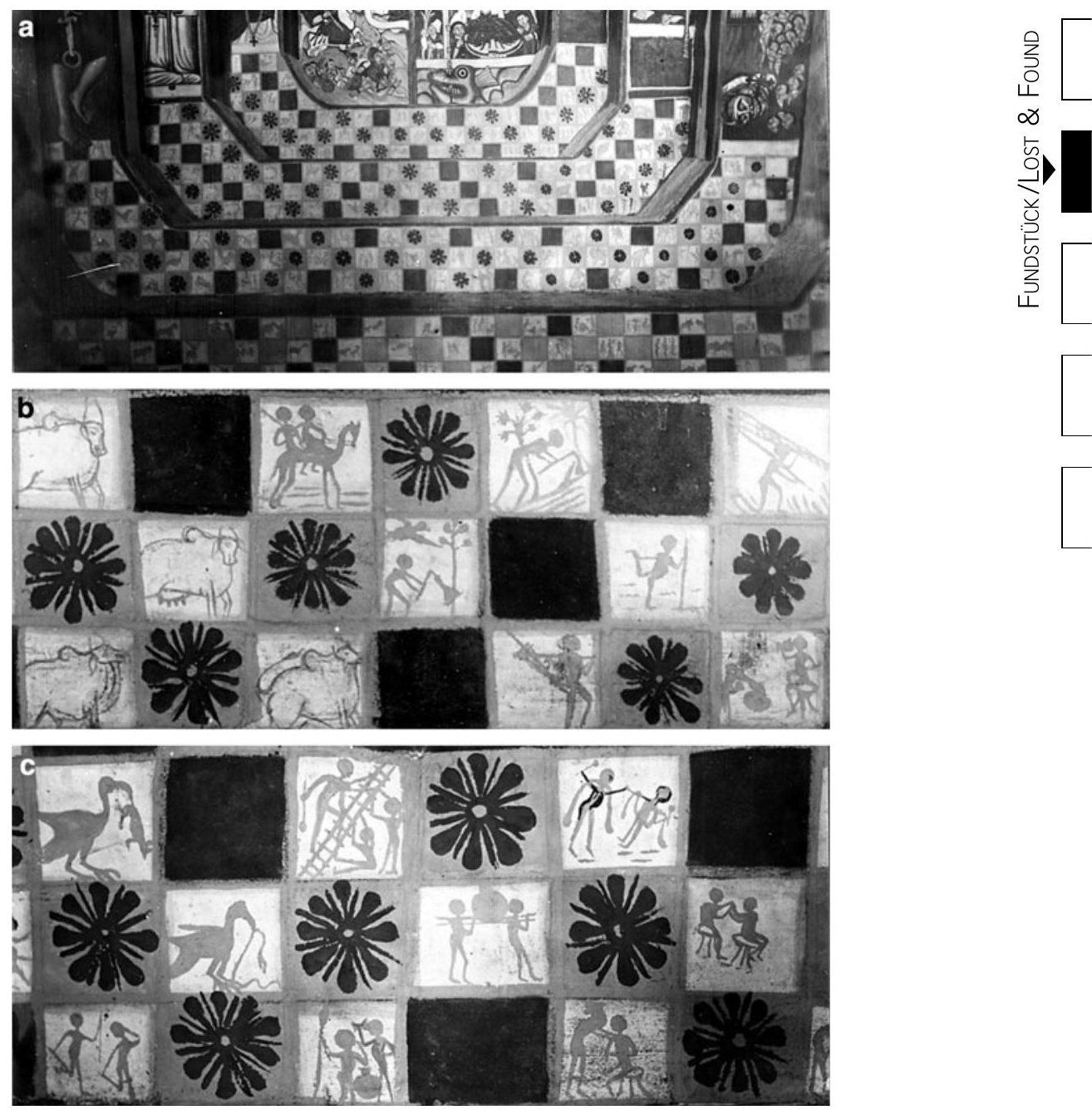

Abb. 4 a Fries des Westors von Zarabruk im Detail (Griaule 1933, Tafel V, Abb. 12), b Silhouetten von Wouddié (Griaule 1933, Tafel VI, Abb. 14), c Silhouetten von Wouddié (Griaule 1933, Tafel VI, Abb. 15)

halbem Weg zwischen fixierter religiöser Tradition und freier Improvisation in einem Spiel subtiler Abstraktion dargestellt sind. ${ }^{11}$ Der Maler Wouddié ist sowohl der Autor der Fresken wie auch der Friese aus der Zarabruk-Kirche in der Nähe von Addiet, die Griaule fotografiert hat. Gleichzeitig hat Wouddié auf Bitten des Ethnologen entsprechende Silhouetten auf europäischem Papier selbst reproduziert.

Obwohl sie dasselbe Material darstellen, sind beide Bildserien dennoch sehr verschieden: fotografische Vergrößerungen der Friese einerseits und einzelne Silhouetten andererseits, die vom einheimischen Maler in isolierter Weise 
a

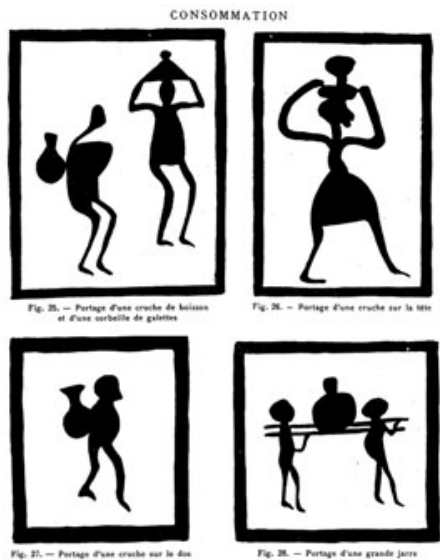

C

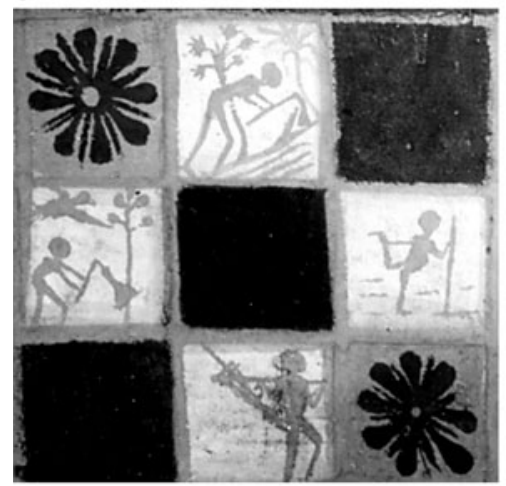

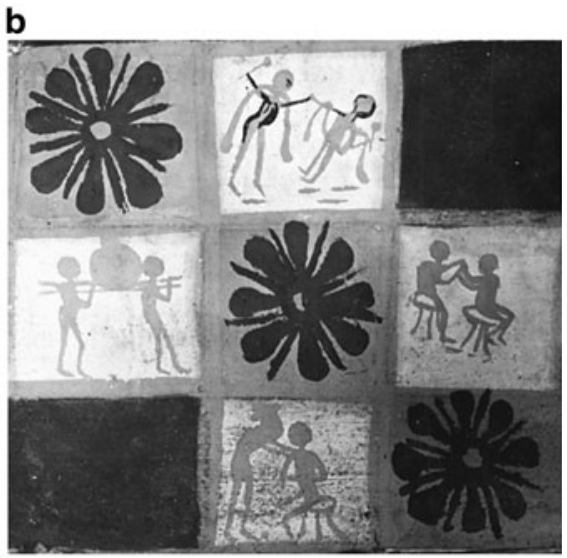

d

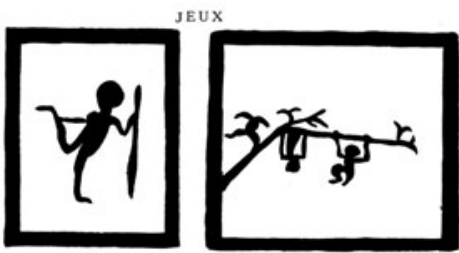

Fe. 6. - Boilerie

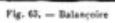

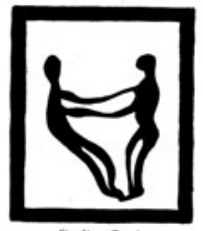

Fie 4t. - Touph

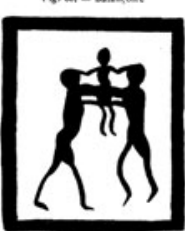

Fis, as. - Bascato

Abb. 5 a Silhouettenmotiv Verbrauch (Griaule 1933: Tafel VII, Abb. 25-28), b Detail des entsprechenden Frieses (Griaule 1933, Tafel VI, Abb. 14), c Detail des Frieses (Griaule 1933: Tafel VI, Abb. 15), d Silhouettenmotiv Spiel (Griaule 1933: Tafel XVII, Abb. 62-65)

gemalt wurden. Der Ethnologe unterwirft letztere seiner eigenen Klassifikation: Angeordnet sind sie nach Techniken des Bauens, der Landwirtschaft, des Verzehrs, des Krieges, der Jagd, des Handels, des Spiels und so weiter. In beiden Fällen stellen die Silhouetten dieselben Alltagstätigkeiten dar. Darunter befinden sich auch elementare Körpertechniken wie das Gehen, Schwimmen, Rennen, Springen, Kämpfen. Gleichwohl muss man den Unterschied zwischen der Verknüpfung der Motive auf den Friesen und der Aneinanderreihung der Einzelsilhouetten durch Griaule beachten (Abb. 5). 
Zwar ging der Gesichtspunkt des „indigenen Handelnden“ zunächst unmittelbar in jenes eigenhändig produziertes Material ein, zugleich wird dieser jedoch verschoben und in einem ihm zum Teil heterogenen Erkenntnisprozess ausgeleuchtet. Jene Silhouetten, die auf dem Fries nebeneinander angeordnet sind und mit abstrakten Motiven wie schwarzen Kästchen oder pflanzartigen Mustern abwechseln, sind in der Darstellungsform Griaules auf verschiedenen Seiten - seiner eigenen Klassifikation folgend - abgedruckt. Die Umrisszeichnungen werden also aus ihrer peripheren Funktion als ornamentaler Rhythmus herausgelöst. Gerade dies aber erlaubt, dem Ethnologen die elementaren Techniken, die für die Godjam-Kultur charakteristisch sind, zu erfassen. ${ }^{12}$ Durch die isolierte und vergrößerte abstrakte Darstellung der Figurenbewegung wird die Verschmelzung zwischen dem Körper und seiner Technik besonders markant hervorgehoben. In der Tat lässt das Silhouettenbild den Menschen und sein Werkzeug völlig homogen werden - ein wenig wie in der Fotografie, deren charakteristische „Entanthropomorphisierung“ oft hervorgehoben wurde. $^{13}$

Durch diese doppelte Dezentrierung und Brechung der indigenen Graphie über die fotografische Reproduktion konnten nun die elementaren Körpertechniken dieser Kultur als solche erfasst werden. Als ob es gerade die Verknüpfung von europäischer Fotografie und afrikanischer Silhouette ermöglicht hätte, die technisch-physische Handlung und die ihr sonst untrennbar anhaftende magisch-religiöse Bedeutung zu zerlegen. Man kann hierzu feststellen, dass sich unter den vielen bis jetzt unveröffentlichten abessinischen Aufnahmen Griaules auch Fotografien finden, die dieselben Körpertechniken im Leben zeigen wie die auf Silhouetten Wouddiés dargestellten - wie hier zum

a

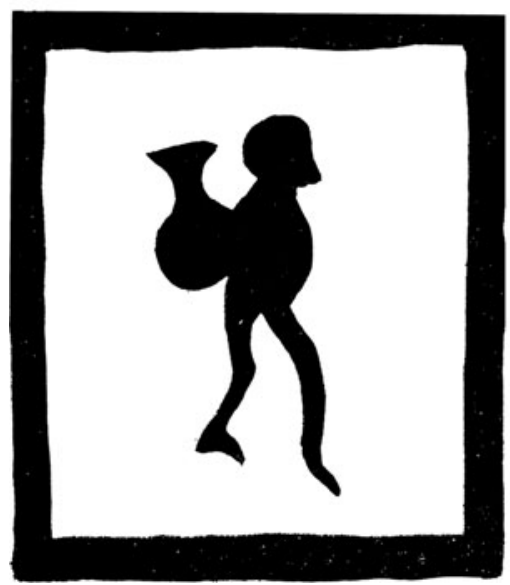

b

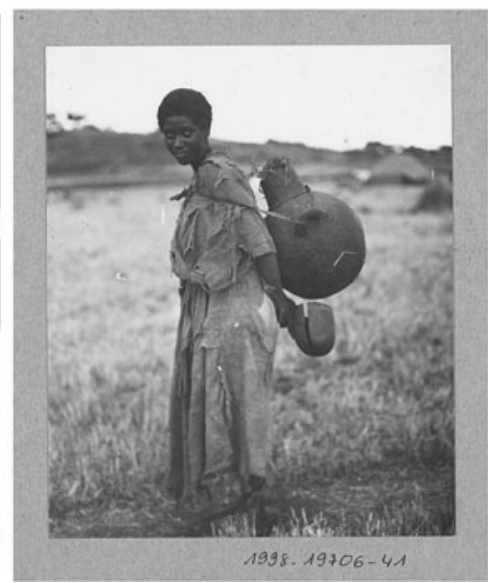

Abb. 6 a Wouddiés Silhouette: Tragen eines Kruges (Griaule 1933: Tafel XVII, Abb. 27), b Griaules Fotografie: Frau (Godjam Addiet) beim Wasserdienst (Sammlung Griaule, Musée Branly, Nr. 1998-19706-41) 
Beispiel das Tragen eines Wasserkruges. Könnte der Ethnologe und Fotograf vom abbessinischen Zeichner das Sehen gelernt haben? (Abb. 6)

Zwar kommt der Begriff der Körpertechnik als solcher in der von Griaule und Mauss gemeinsam gezeichneten - aber sehr wahrscheinlich allein von Ersterem verfassten - Einleitung zur abessinischen Publikation nicht vor. Da aber Griaule seinem Mentoren direkt aus dem Feld Fotos zu schicken pflegte, kannte Mauss diese Silhouetten zum Teil seit der Expedition. ${ }^{14}$ Außerdem hatte er dieses Bildmaterial als Ganzes - im Rahmen der gemeinsamen Publikation - noch im Jahr vor seinem Vortrag von 1934 vor Augen gehabt. Insofern hat die afrikanische Darstellungsweise einen bedeutenden Anteil an der fotografischen Verdichtung dessen gehabt, was schließlich techniques $d u$ corps genannt werden sollte.

\section{Danksagung}

Für Anregungen bei der Diskussion dieses Beitrags möchte ich mich insbesondere bei Michael Cowan (Montreal), Ines Lindner (Kiel) sowie bei den Kollegen des Internationalen Forschungszentrums für Kulturwissenschaften in Wien Helmut Lethen, Lutz Musner und Jan von Brevern bedanken.

\section{Anmerkungen}

1 Ich zitiere hier wie im Folgenden die deutsche Übersetzung von Axel Schmalfuß des Vortrags, die leicht modifiziert wurde, vgl. Mauss 1950 [1936]: 367.

2 Vgl. Brief von Mauss an Ignace Meyerson, zitiert in Fournier 1994: 529.

3 Vgl. unter den wenigen Ausnahmen: Schüttpelz 2002: 161 f.; Leveratto 2006; ferner auch Latour 2005: 210, der den Passus aber unkommentiert zitiert.

4 "Verfremdung" wird hier verstanden im Sinne des von Ginzburg verwendeten italienischen Begriffs straniamento (Russ. ostranjenje, franz. estrangement), vgl. Ginzburg 1999: 11.

5 Mauss' Bemerkung befindet sich hier ganz in der Nähe von Beobachtungen, die in essayistischen Schriften über das Kino der Zeit beispielsweise bei Joseph Roth oder Siegfried Kracauer zu finden sind.

6 Mauss [1947] 1967: 3. In seinen nächsten Vorlesungen wird deutlich, dass der Begriff „Körpertechniken“ dazu beitragen sollte, den Menschen in erster Linie als „rhythmisches Tier" (ebd.: 85) zu definieren. Er bedingt einen möglichen Paradigmenwechsel, dessen epistemologische Tragweite erst von der Paläo-Anthropologie erkannt wurde, siehe LeroiGourhan 1965.

7 Zu diesen Urtechniken zählt Mauss: Geburt, Schlaf, Ausruhen, Bewegung - Gang, Lauf, Tanz usw., Körperpflege, Verzehr bis hin zum Geschlechtsverkehr.

8 Einer möglichen Beziehung dieser Zuspitzung mit der ethnologischen Mission in Marokko im Auftrag des Instituts für Anthropologie wäre noch nachzugehen: vgl. den „Rapport présenté à l'Institut français d'anthropologie“ (1930), zitiert als [Sémites et africains au Maroc], in Mauss 1974: $562 \mathrm{ff}$.

9 Vgl. Griaule, 1933; ferner Griaule 1991: 132.

10 Einige der Graffiti wurden als Illustration des Essays von Georges Bataille: „L'art primitif“ verwendet, das $1930 \mathrm{im}$ 2. Jahrgang der Zeitschrift Documents erschien.

11 Vgl. Griaule 1933: 6; sowie ferner: „Ces œuvres relèvent d'une technique très différente de celle qui est employée pour les autres peintures, où l'artiste, prisonnier de règles étroites, 
travaille sans regarder la nature. Là au contraire, il prend ses modèles autour de lui et reproduit avec exactitude des gestes, des expressions, des détails typiques de la vie courante." (ebd.).

12 So können die Silhouetten als visueller Katalog von Alltagshandlungen und -techniken verstanden werden.

13 Vgl. beispielsweise Kracauers Fotografieauffassung, der sich dabei auf Proust beruft (Kracauer 1960, 14 f.).

14 Vgl. unter anderem Griaules Brief an Mauss vom 19. Februar 1929, IMEC (Institut Mémoires de l'Edition Contemporaine), MAS, 5.45.

\section{Literatur}

Braun, Marta, 2006. Marey, Muybridge, le sport et la race. In: E. J. Marey, Actes du colloque du centenaire, Dominique de Font-Réaulx, Thierry Lefebvre und Laurent Mannoni, Hg., Paris: Arcadia Editions, 69-78.

Comte, M./Regnault Félix, 1896. Marche et course en flexion, Bulletins de la Société d'anthropologie de Paris, IV, Bd. 7, 337-341.

Fournier, Marcel, 1994. Marcel Mauss. Paris: Fayard.

Ginzburg, Carlo 1999. Holzaugen. Über Nähe und Distanz. Berlin: Wagenbach.

Griaule, Marcel, 1933. Silhouettes et graffiti abyssins. Introduction de Marcel Mauss. Paris: Larose. Griaule, Marcel, [1934] 1991. Les flambeurs d'hommes. Paris: Berg International.

Kracauer, Siegfried, 1960. Theory of Film. The Redemption of Physical Reality. New York: Oxford University Press.

Leroi-Gourhan, André, 1965. Le geste et la parole II: la mémoire et les rythmes. Paris: Albin Michel. Latour, Bruno, 2005. Reassembling the Social. An Introduction to Actor-Network-Theory. Oxford: Oxford University Press.

Leveratto, Jean-Marc, 2006. Lire Mauss; Les techniques du corps au cinéma, Le Portique, No 17 Marcel Mauss et les techniques du corps, http://leportique.revues.org [Zugriff am 25.01.2010].

Mauss, Marcel, [1936] 1950. Les techniques du corps (Journal de Psychologie, XXXII, no 3-4, 1936). In: ders.: Sociologie et anthropologie. Paris: Presses Universitaires de France, 363-386.

Mauss, Marcel, [1947] 1967. Manuel d'ethnographie. Paris: Payot.

Mauss, Marcel, [1930] 1968. Les civilisations: éléments et formes (1930). In: ders., Essais de sociologie. Paris: Le Seuil, 231-252.

Mauss, Marcel, [1930] 1974. Sémites et africains au Maroc. In: ders., Euvres 2. Représentations collectives et diversité des civilisations, Victor Karady, Hg., Paris: Editions de Minuit, 562-567.

Mauss, Marcel, [1936] 1975. Die Techniken des Körpers. In: Ders., Soziologie und Anthropologie, hrsg. von W. Lepenies und H. Ritter, 2. Bd. München: Hanser, 199-220.

Schüttpelz, Erhard, 2002. Der Fetischismus der Nationen und die Durchlässigkeit der Zivilisation. Globalisierung durch technische Medien bei Marcel Mauss (1929). In: Stefan Andriopoulos und Bernhardt Dotzler, Hg., 1929. Beiträge zur Archäologie der Medien. Frankfurt a. M.: Suhrkamp, 158-172.

\section{Philippe Despoix}

Département de Littérature comparée

Université de Montréal

C.P. 6128 succursale Centre-ville

Montréal (QC) H3C 3J7

Canada

E-Mail: philippe.despoix@umontreal.ca 